\title{
Meta
}

Journal des traducteurs

Translators' Journal

\section{VAN STERKen burg, P. (compilateur) (2003) : A Practical Guide to Lexicography, Amsterdam / Philadelphie, John Benjamins Pulblishing Company, XII + 460 p.}

\section{Henri Béjoint}

Volume 49, numéro 4, décembre 2004

URI : https://id.erudit.org/iderudit/009802ar

DOI : https://doi.org/10.7202/009802ar

Aller au sommaire du numéro

Éditeur(s)

Les Presses de l'Université de Montréal

ISSN

0026-0452 (imprimé)

1492-1421 (numérique)

Découvrir la revue

Citer ce compte rendu

Béjoint, H. (2004). Compte rendu de [VAN STERKENBURG, P. (compilateur) (2003) : A Practical Guide to Lexicography, Amsterdam / Philadelphie, John Benjamins Publishing Company, XII + 460 p.] Meta, 49(4), 952-958.

https://doi.org/10.7202/009802ar d'utilisation que vous pouvez consulter en ligne.

https://apropos.erudit.org/fr/usagers/politique-dutilisation/ 
van Sterkenburg, P. (compilateur) (2003): A Practical Guide to Lexicography, Amsterdam / Philadelphie, John Benjamins Publishing Company, XII + 460 p.

Piet van Sterkenburg présente ici 29 contributions de 31 auteurs, y compris lui-même, qui constituent un guide pratique de lexicographie comportant 387 pages de texte, 30 pages de glossaire, 20 pages de bibliographie en une seule liste regroupant les dictionnaires et les sources métalexicographiques et 18 pages d'index. Comme les autres volumes dans cette série dirigée par Marie-Claude L’Homme, Ulrich Heid et Juan Sager ( Terminology and Lexicography Research and Practice»), le livre est très bien présenté, très clair, très agréable à lire. Il est dommage qu'aucun détail ne soit donné sur les auteurs, pas même l'institution à laquelle ils appartiennent; j'en connais un certain nombre, mais le livre ne m'est d'aucun secours pour les autres. Or il s'agit d'une indication particulièrement importante en métalexicographie: les auteurs ont-ils une expérience de lexicographie pratique? Laquelle? Il est tout de même clair qu'il y a une forte représentation d'auteurs néerlandophones'.

Le texte, entièrement en anglais, est divisé en deux parties: «I. The forms, contents and uses of dictionaries", et "II. Linguistic corpora (databases) and the compilation of dictionaries». Chaque partie est ensuite divisée en chapitres et chaque chapitre constitué d'articles (de 2 à 6 selon les chapitres), chaque article couvrant de 8 à 25 pages, avec une moyenne de 13 pages. La répartition des articles entre les deux parties obéit à une logique qui n'est pas évidente pour le lecteur, comme on le verra ci-dessous.

Les dimensions du livre, les titres de certains articles et les noms de certains des auteurs ne peuvent qu'allécher l'amateur de dictionnaires. Les objectifs sont clairement énoncés dès la préface: il s'agit d'abord de tracer les grandes lignes des théories linguistiques dans lesquelles s'inscrit l'activité du lexicographe, et de décrire les méthodes utilisées naguère et maintenant pour compiler un dictionnaire. Il s'agit aussi, dans la deuxième partie, d'expliquer comment la lexicographie utilise désormais les outils électroniques. Le livre est né, nous explique-t-on dans la préface, d'une constatation de Juan Sager en 1993: il n'existait pas de guide pratique utilisable pour initier des étudiants à la lexicographie. Le projet aura donc mis dix ans pour mûrir, pour aboutir à ce volume placé clairement sous le patronage de la linguistique et qui ambitionne de couvrir l'essentiel des questions que peuvent se poser les apprentis lexicographes et toutes les personnes qui s'intéressent à la façon dont les dictionnaires sont fabriqués.

Dans le cas d'un livre constitué d'articles écrits par des auteurs différents, il est difficile pour le recenseur d'éviter un passage en revue des articles. C'est ce que je me propose de faire, en leur donnant plus ou moins d'importance en fonction de mes goûts personnels et de mes préoccupations du moment. Que les auteurs rapidement évoqués me pardonnent: la qualité de leur contribution n'est pas forcément en rapport direct avec la longueur de mon commentaire.

La première partie ( «The forms, contents and uses of dictionaries») comporte trois chapitres. Le chapitre 1 («Foundations») commence par un article de Piet van Sterkenburg lui-même, «The «dictionary»: Definition and history». Il y traite, dans l'ordre, la définition de dictionnaire et l'histoire du genre, en se concentrant quasi exclusivement sur le dictionnaire monolingue général, qui en est le prototype. Rien de très nouveau, mais c'est évidemment un chapitre indispensable dans un manuel. Dans le deuxième article (1.2), «Source material for dictionaries ", Frantisek Cermak, dont on connait les écrits sur la phraséologie, explore les différentes façons pour un lexicographe de rassembler les données nécessaires à la compilation du dictionnaire. Il compare le Czech National Corpus et le British National Corpus, qui ont tous les deux environ 100 millions de mots (au moment de la comparaison) mais qui ont des contenus différents. Par exemple, la proportion de textes provenant de journaux n'est pas la même, ce qui interroge sur la représentativité d'un corpus pourtant considérable. Beaucoup de sources qu'il cite sont en tchèque, et ne seront donc pas accessibles 
à tous, mais je trouve très bien qu'un manuel de lexicographie invite ses lecteurs à lire d'autres langues que l'anglais. "Uses and users of dictionaries", la troisième contribution de ce chapitre (1.3), est de Paul Bogaards, dont les écrits sont bien connus dans ce domaine. Il y passe en revue les différents types d'études des utilisateurs et des utilisations des dictionnaires et redit ici ce qu'il a dit dans d'autres publications: c'est en fonction de ces utilisateurs et de ces utilisations que les dictionnaires doivent être conçus. Il a raison, au moins tant que l'on parle de dictionnaires «fonctionnels», même s'il ne faut pas oublier que les dictionnaires peuvent aussi se donner pour objectif de donner une image du lexique d'une langue, auquel cas les considérations sur l'usage qui en sera fait sont moins importantes. L'article suivant («Types of articles, their structure and different types of lemmata», 1.4), signé Rufus Gouws, est assez technique et pas toujours facile à lire. Il reprend utilement, entre autres choses, la distinction entre articles à niches ( "niched entries») et articles à nids (nested entries). «Dictionary typologies: A pragmatic approach» (1.5), de Piet Swanepoel, synthétise les nombreuses typologies existantes. L'article comporte des illustrations (qui incidemment auraient pu être plus nombreuses dans l'ensemble du livre $)^{2}$. Il invite plusieurs fois le lecteur à analyser ces illustrations, comme il est normal dans un manuel, même s'il le fait de manière un peu déroutante: "Study Example 6», sans autre indication sur ce qu'il s'agit d'observer, ni solution, ou «Consider Example 5» sans explication supplémentaire. Ceci produit sur le lecteur une impression bizarre dans la mesure où c'est le seul article qui procède de cette manière. L'article ne fait pas mention du dictionnaire Macmillan, qui n'était sans doute pas sorti au moment où l'auteur écrivait - illustration des nuisances engendrées par les délais de publication. L'article a aussi des renvois à d'autres articles, qui montrent que Piet Swanepoel est un des rares contributeurs à avoir pleinement pris acte des objectifs du volume.

Le chapitre 2 («Descriptive lexicography») commence par «Phonological, morphological and syntactic specifications in monolingual dictionaries» (2.1) de Johan de Caluwe et Ariane van Santen, qui n'est pas très convaincant. Entre les confusions terminologiques ( lemma» pour «entry», p.71 et passim), les renvois qui ne renvoient nulle part («see Section $2.9 »$, p.75; «see $\$ 3.2 »$, p.76), l’imprécision des références ( Longman», «the Oxford», «Chambers», «Collins» - sans doute le COBUILD - et «OED»), les lourdeurs ( productive affixes that can produce new words», p.73) et les passages consacrés à des explications basiques sur la grammaire de l'anglais, le lecteur a du mal à suivre. En fait, on a l'impression d'un article qui aurait été écrit pour une autre destination, et trop vite adapté pour figurer dans ce volume. L'article "Meaning and definition»(2.2), signé Dirk Geeraerts, est d'un autre tonneau. Il s'agit d'une très courte mais stimulante introduction aux problèmes généraux de la définition lexicographique, à laquelle on ne peut reprocher que d'être un tantinet trop générale et superficielle, et qui aurait donc besoin d'être complétée, par exemple par Landau (2001) pour les considérations d'un praticien de la lexicographie. Le titre de l'article 2.3, «Dictionaries of proverbs», rédigé par Stanislaw Predota, surprend ici: pourquoi un article sur les dictionnaires de proverbes, alors qu'il n'y en a pas sur les dictionnaires étymologiques, ou sur les dictionnaires pour apprenants, ou d'argot, tous plus «centraux» que les dictionnaires de proverbes? Et pourquoi à cet endroit du manuel, alors qu'il y a un chapitre 3 intitulé «Special types of dictionaries»? L'article, très court, utilise les notions de proverbe, d'adage, de dicton et autres mais ne les définit pas. L'article 2.4 (Igor Burkhanov) porte un titre qui laisse craindre l'hétérogénéité du contenu: "Pragmatic specifications: Usage indications, labels, examples; dictionaries of style, dictionaries of collocations ». Comme il n'est pas découpé en parties et que le développement n'est pas d'une clarté aveuglante, il est difficile à utiliser. Il cite des dictionnaires par des abréviations («COB3», «ALDCE3», «RHUD2», «WNID3», «LDOPV», «LDPHV», etc.) qui ne sont déchiffrables que par des spécialistes de la question (et encore!), et qui renvoient à une bibliographie (c'est le seul article de tout le recueil à avoir une bibliographie séparée, uniquement 
d'ailleurs pour les dictionnaires) où les ouvrages sont classés par titre et non par abréviation. En outre, ce ne sont pas les mêmes abréviations que celles qui sont utilisées dans d'autres articles. Le passage sur les dictionnaires de collocations est beaucoup trop court (quelques paragraphes) et n'éclaire pas le lecteur sur les différences entre expression idiomatique et collocation. Il faudra pour cela voir l'article 7.4 (voir ci-dessous), auquel celui-ci ne renvoie pas. «Morphology in dictionaries» (2.5), de Johan de Caluwe et Johan Taeldeman, promet de faire double emploi avec 2.1 mais en fait il parle de manière assez intéressante des problèmes posés par le traitement des dérivés et des composés. L'article de Piet van Sterkenburg ( Onomasiological specifications and a concise history of onomasiological dictionaries », 2.6) constitue un apport précieux à cause de la rareté des références disponibles sur ce vaste sujet. L'auteur y explique le travail de classification sémantique qui est fait à l'«Institute for Dutch Lexicology» de Leiden dans le cadre de la préparation du Algemeen Nederlands Woordenboek, travail destiné à permettre à l'utilisateur de retrouver les unités lexicales dont il a besoin, et de comparer celles qui sont sémantiquement proches. L'histoire des dictionnaires onomasiologiques est abordée brièvement à la fin de l'article. Incidemment, l'auteur considère comme beaucoup d'autres que l'onomasiologie est l'exact inverse de la sémasiologie, alors que les choses, me semble-t-il, sont plus complexes ${ }^{3}$.

Le chapitre 3 ( Special types of dictionaries») commence par un article de Mike Hannay sur "Types of bilingual dictionaries» (3.1), assez succinct, mais qui contient les considérations essentielles. Le thème de la lexicographie spécialisée et des dictionnaires spécialisés (3.2) est traité par Lynne Bowker, avec sa clarté habituelle. L'ensemble reste toutefois un peu abstrait: sa typologie, par exemple, évoque des dictionnaires spécialisés pour «nonnative speakers» (p. 156), ou spécialisés dans la "production» (p. 157), types logiques en théorie mais dont elle ne cite aucune réalisation. Par contre, elle ne fait qu'évoquer les distinctions subtiles établies par certains entre lexicographie, lexicographie spécialisée et terminographie. Elle décrit une procédure de compilation qui concilie les exigences de la terminologie traditionnelle et les tendances de la terminologie moderne: d'abord onomasiologique pour délimiter les contours du domaine, elle est ensuite sémasiologique, à partir des textes du corpus, pour identifier les termes et les traiter (même la définition du terme est extraite du contexte), et revient à l'onomasiologie pour la présentation des données: «one entry is prepared for each concept» (p. 163), sans expliquer comment on passe du concept identifié dans la première étape au terme repéré dans la deuxième puis au concept traité dans la troisième. L'article se termine par un bref passage sur les autres types de dictionnaires spécialisés, en particulier les dictionnaires de variétés régionales, si bref qu’on ne voit pas bien pourquoi il est là, si ce n'est pour satisfaire le désir du compilateur de «couvrir» aussi cet aspect de la lexicographie.

Globalement, cette première partie n'aura tenu que partiellement ses promesses. Elle évoque certes des approches théoriques de la lexicographie basées sur la linguistique, mais elle ne peut prétendre être une introduction à l'ensemble des problèmes théoriques de la discipline: elle ne dit rien des travaux des théoriciens de la lexicographie, Wiegand, par exemple. Elle n'apporte aucune aide sur le problème de l'identification de l'unité lexicographique, ne dit rien ou presque sur la sémantique des cadres ou sur la sémantique du prototype, ou sur les travaux de Wierzbicka ou de Mel'čuk.

La deuxième partie ( II. Linguistic corpora (databases) and the compilation of dictionaries») comporte quatre chapitres. Le premier («Corpora for dictionaries») commence par deux articles de John Sinclair. Dans «Corpora for lexicography» (4.1), il prodigue des conseils à ceux qui veulent construire un corpus devant servir de base à un dictionnaire. Venant de quelqu'un comme John Sinclair, avec son expérience, ces conseils sont très utiles. Curieusement, l'auteur n'y aborde pas la question de savoir si un corpus lexicographique doit être fermé ou ouvert. Le deuxième article de John Sinclair («Corpus processing», 4.2) est 
un plaidoyer pour des options qui s'écartent délibérément des pratiques les plus courantes, dont il dit qu'elles ont été imposées aux linguistes par des gens qui n'en sont pas: il plaide pour un corpus de texte brut, qui n'est ni une archive ni une base de données, et pour des annotations automatiques, des opérations en ligne et des data streams séparés. Sinclair accuse solennellement le clan des «annotateurs» de corrompre les textes (ils auront à rendre compte de leurs forfaits, dit-il, au même titre que ceux de nos ancêtres qui ont mutilé les statues pour cacher à la vue les parties qu'ils jugeaient honteuses) et de produire des corpus qui empêchent les linguistes de voir des phénomènes linguistiques, éventuellement importants, qui n'ont pas été prévus par leurs systèmes d'annotations. Il accuse le TEI d'être coûteux, inutile et même gênant. Le ton est vibrant, l'ensemble très stimulant, et de très haut niveau, peut-être trop pour des lexicographes débutants. Mais, dit Sinclair avec une sagesse qui ne souffre pas de contestation, «Any new venture would be wise to start with the best practice of the future, not of the past» (p. 191). «Multifunctional linguistic databases: Their multiple use» (4.3), de Truus Kruyt, aborde le problème de la construction de vastes ressources langagières à partager entre divers projets, y compris, mais pas exclusivement, lexicographiques, et regrette que la communication et la collaboration entre lexicographes et spécialistes d'autres disciplines ne soient pas aussi intenses qu'il serait souhaitable. Il mentionne évidemment le TEI parmi les outils qui facilitent cette collaboration (le contraste avec ce que dit Sinclair juste avant est saisissant). L'article 4.4 («Lexicographic workbench: A case history» (Daniel Ridings) détaille le Manuel de style du «Buro van die Woordeboek van die Afrikaanse Taal» (WAT). Il n'a rien de très original, mais son côté pratique sera utile à des lexicographes travaillant sur d'autres projets.

Le chapitre 5 ( «Design of dictionaries») commence par un article intitulé «Developments in electronic dictionary design » (Lineke Oppentocht et Rik Schutz). L'article est bien écrit et plein d'informations, mais ne donne guère de références. "Linguistic corpora (databases) and the compilation of dictionaries» (5.2), de Krista Varantola, ne traite pas exactement le sujet que son titre annonce, mais contient des considérations intéressantes sur les besoins des utilisateurs, la préface des dictionnaires, l'utilisation des abréviations et des symboles, la typographie et la mise en page, etc. Les références y sont données sous forme de notes, contrairement aux autres articles du livre. L'article 5.3, "The design of online lexicons", de Sean Michael Burke, n'est pas sans intérêt, mais on a du mal à voir sa place dans l'ensemble.

Le chapitre 6 («Realisation of dictionaries») commence par un article sur «The codification of phonological, morphological, and syntactic information» (6.1), de Geert Booij, qui est sans doute intéressant pour quiconque veut en savoir plus sur la grammaire du néerlandais mais n'a guère de rapport avec cette partie de l'ouvrage consacrée théoriquement aux questions de la réalisation des dictionnaires en liaison avec le corpus. "The production and use of occurrence examples» (6.2) de John Simpson, est précieux par l'expérience de son auteur. Clair et pratique, il sera très utile, même s'il n'a que peu de rapports avec le corpus. Il est le seul article de tout le volume à proposer de vrais exercices, d'ailleurs fort bien faits, au lecteur. L'idée était bonne pour un livre qui se présente comme un manuel, et on se demande pourquoi elle n'a pas été utilisée dans les autres articles. "The codification of semantic information» (6.3) de Fons Moerdijk complète utilement l'article de Geeraerts évoqué ci-dessus, auquel il renvoie explicitement (de même qu'à l'article de Verkuyl, Janssen et Jansen, p. 290, en se trompant sur l'ordre des auteurs), pratique suffisamment rare dans ce volume pour être signalée. L'article 6.4 ( «The codification of usage by labels »), de Henk Verkuyl, Maarten Janssen et Frank Jansen, visiblement rédigé à la hâte, est très difficile à suivre, indûment théorique et me semble peu utile. L'article suivant, «The codification of etymological information» (6.5) de Nicoline van der est très court, mais aborde l'essentiel des questions liées à l'inclusion de l'étymologie dans les dictionnaires 
généraux. Il est dommage, là aussi, que les nombreux exemples donnés soient attribués à des dictionnaires impossibles à identifier (Chambers, Longman, Merriam-Webster, Duden, Wahrig, Larousse, etc.).

Dans le septième chapitre («Examples of design and production criteria for major dictionaries»), le premier article («Examples of design and production criteria for bilingual dictionaries », 7.1), de Wim Honselaar, aborde la question de la préparation des bilingues. Le texte est utile, même s'il n'est qu'un survol des questions à résoudre. Il renvoie de manière énigmatique au «Coursebook» (p. 323), qui a dû être le titre du livre à un certain stade de sa préparation. Martin et van der Vliet traitent assez en détail de la préparation des dictionnaires spécialisés («Design and production of terminological dictionaries», 7.2). Une partie de ce qui est dit ici, fondamental en terminographie, par exemple les différences principales entre terminographie et lexicographie, aurait peut-être été mieux placée dans l'article de Bowker (3.2). Les auteurs décrivent des étapes de la rédaction d'un recueil de termes dont ils disent qu'elles sont trop souvent passées sous silence dans la littérature: la modélisation du domaine (domain modelling), la modélisation des données (data modelling) et la modélisation des concepts (concept modelling). Ils placent clairement les trois opérations sous le chapeau de la modélisation cognitive, qui est à leurs yeux ce qui manque le plus à la pratique terminographique. Ils évoquent également les bénéfices que peut tirer le terminographe d'une classification plus fine des termes, qu'ils proposent d'entreprendre par l'intermédiaire des cadres de Minsky et de Fillmore. Il n'est pas certain que ce soit la seule théorie qui permette cette classification, mais on sera d'accord sur le fait qu'une classification sémantique fine permet l'amélioration des définitions et facilite l'accès aux termes. L'article 7.3 («Design and production of monolingual dictionaries»), de Ferenc Kiefer et Piet van Sterkenburg, reprend utilement les étapes de la conception d'un dictionnaire, un peu à la manière de Landau (2001), avec quelques renvois (trop peu nombreux, et parfois fautifs ou incomplets) aux autres articles du livre. Enfin, le dernier article (7.4), «Towards an « deal» Dictionary of English Collocations », signé Stefania Nuccorini, examine très en détail plusieurs dictionnaires de collocations de l'anglais.

Globalement, le lecteur de cette deuxième partie pourra rester un peu sur sa faim, dans la mesure où le contenu n'est pas à la mesure de ce que le titre annonce: beaucoup d'articles n'ont pas grand chose à voir avec les corpus et leurs utilisations dans la préparation des dictionnaires.

Le glossaire, normalement très utile, n'est pas exempt de reproches. Par exemple, Bi-directional y est défini par «being reception and production-oriented» (je n'ai jamais rencontré ce sens-là, mais il est peut-être usité), et Bidirectional dictionary (avec la même majuscule mais sans trait d'union) par «a bilingual dictionary that provides translation equivalents of two languages both for production and reception»; Genus par «a group of lexemes within a family that consists of a number of similar or closely related species» (où species côtoie bizarrement lexeme); Lexeme par «the smallest distinctive unit in the lexicon or vocabulary of a language which is mostly interpreted as a combination of a form with a meaning» (c'est exactement ce que Cruse 1986 appelle lexical unit), avec un renvoi à lexical item et lexical unit, dont les entrées ne font que renvoyer à lexeme; Slang par «a usage label ...». DTD, head (d'un composé), TEI et d'autres sont absents. La bibliographie comporte beaucoup d'erreurs et de références incomplètes.

Ce livre promettait beaucoup, par son volume et la qualité de ses auteurs, mais il ne tient pas toutes ses promesses. D'abord, on peut regretter l'absence de certains aspects de l'activité lexicographique, le rôle social du dictionnaire, par exemple, ou la nature de l'unité de traitement lexicographique, notion pourtant fondamentale, ou encore la rareté des allusions aux travaux des germanophones ou des francophones. Mais admettons qu'un manuel ne peut pas tout traiter. Ensuite, il y a trop d'erreurs matérielles qui nuisent au confort du lecteur et à son adhésion. Il y a des références introuvables: Reichmann 1989 (p. 47), 
Hartmann 1983 (p. 49), Selbridge 1983 (p. 60), Scheinmann 1989 (p. 62), Morris and Morris 1975 (p. 65), « contemporary Van Dale» (1996) (p. 72), «'larger Van Dale' (1999)» (p. 72), Cruse 1984 (p. 110), Webster 1978 (p. 128), van Sterkenburg 1995 (p. 131), Bernstein 1976 (p. 134), Püschel 1994 (p. 142), Schaller 1985 (p. 143), Agricola 1977 (p. 143), Bogaards to appear (p. 153), Illustrated Dictionary of Computing (p. 157), Tognini Bonelli 2001 (p. 185), Jelinek 1985 (p. 189), Curry 1996 (p. 240), Kick \& Henry 1988 (p. 243); American Heritage 1994 (p. 249), Webster's (p. 262), Milroy and Milroy 1990 (p. 311), van den Baars Comprehensive Dutch-Russian Dictionary (p. 328), Schaetzen 1998 (p. 349), Martin 1988 (p. 353), Sinclair, Kruyt \& Ridings (p. 353), A Deskbook of Most Frequent English Collocations (p. 366). Il y a aussi des références incomplètes ou fautives: Swanepoel \& Van der Poel «to appear» dans le texte (p. 44) mais 2001 dans la bibliographie; Wright's English Dialect Dictionary 1898/1905 dans le texte (p. 59) mais 1961 dans la bibliographie; Cassell's Modern Guide to Synonyms \& Related Words attribué à Partridge 1961 (p. 63); Norris \& Norris 1971 (p. 65) mais 1975 dans la bibliographie; Cresswell \& McDavid 1983 (p. 106) mais 1993 dans la bibliographie; Morrison et al. (n.d.) (p. 178) est 2000 dans la bibliographie (aussi p. 211); «Unknown? 1983 » (p. 240 et 244); Marchand 1960 est qualifié de «wordbook» (p. 241); Strauss 1998 (p. 366) est 1994 dans la bibliographie; Correas 1627 (p. 366) est 1927 dans la bibliographie; Biber 1999 (p. 368) est Biber et al. dans la bibliographie. Globalement, il y a une utilisation anarchique des a, b, c, etc. pour distinguer les publications d'un même auteur une même année. Il y a enfin des erreurs typographiques en nombre excessif: j'en ai compté au moins 80. Beaucoup de ces fautes sont des séparations intempestives d'un mot et de sa terminaison du pluriel, ce qui laisse penser à un défaut de logiciel de l'imprimeur.

Enfin et surtout, l'ensemble manque de maîtrise. Le problème de tous les ouvrages composés de contributions d'auteurs différents, surtout s'ils entendent couvrir l'essentiel d'un sujet pour servir de manuel, est que chaque contributeur rédige son article dans une ignorance plus ou moins grande de ce qui sera proposé par les autres. Ce problème peut être en partie résolu par le compilateur, s'il assure la cohérence de l'ensemble, ménage une progressivité, harmonise les contributions a minima (plans, notes, modes de référence et autres détails techniques), s'il ajoute des commentaires, des renvois, s'il vérifie toutes les références, s'il enlève les redites, etc. Cela ne semble pas avoir été le cas ici. On voit donc dans chaque article ou presque des points qui auraient mérité un traitement de la part du compilateur mais qui n'en ont pas fait l'objet. Il y a des différences de style et de technique: certains articles ont des notes, d'autres pas; un article a une bibliographie, mais tous les autres rassemblent leurs références dans une seule bibliographie en fin d'ouvrage; tel article a des exercices, les autres pas, etc. Il y a de nombreuses redites, par exemple à propos des notions de macrostructure / microstructure, de sémasiologie / onomasiologie etc., voire des contradictions entre différents articles. On note aussi des usages terminologiques qui ne correspondent pas aux définitions des termes dans le glossaire ${ }^{4}$. Il y a enfin de grosses différences de niveau de spécialisation: certains articles sont très élémentaires et d'autres beaucoup plus difficiles.

Globalement, il est clair que tout métalexicographe se doit de consulter ce nouvel ouvrage, qui s'inscrit dans la lignée des introductions générales à la lexicographie, et qui ajoute donc à la panoplie des manuels désormais disponibles. On pourra y voir un complément intéressant à Svénsen (1994) et à Landau (2001), par exemple, dans la mesure où les auteurs sont souvent ici des linguistes plus, ou autant, que des lexicographes. On y trouvera beaucoup d'informations, et certains articles remarquables, à des degrés divers et pour des raisons diverses (Geeraerts, Sinclair, Nuccorini). Il est dommage qu'un travail de compilation trop superficiel n'ait pas permis d'éliminer les articles les moins intéressants, de vérifier toutes les références, de jeter des ponts entre les textes et globalement d'harmoniser les contributions pour en faire la véritable somme qu'un tel livre aurait pu être.

HENRi BÉJOINT 


\section{NOTES}

1. Je trouve, ou imagine, une bonne dizaine de Hollandais, trois Anglais, deux Sud-Africains, un Tchèque, un Belge, une Finlandaise, un Hongrois, une Italienne, et quelques autres.

2. Malheureusement les illustrations y sont référencées de manière très imprécise: «Webster's 2nd ed.» (p. 50), «Webster New International Dictionary 2nd ed., 1950» (qui n'existe pas dans la bibliographie), «OED» (qui n'existe pas dans la bibliographie non plus) sans indication de l'édition (p. 54), «Webster's Third» (p. 56).

3. En gros, le sémasiologue va du signifiant au signifié, et reste donc dans le signe, alors que l'onomasiologue va du conceptuel, ou même du référentiel, au linguistique sauf à considérer que l'on part du signifié, ce qui semble bien difficile avant même l'identification d'un signe.

4. Seul Sinclair (p. 178) fait explicitement référence au glossaire du recueil.

\section{RÉFÉRENCES}

Cruse, D.A. (1986) : Lexical Semantics, Cambridge, Cambridge University Press.

Landau, S. (2001): Dictionaries, The Art and Craft of Lexicography, Cambridge, Cambridge University Press.

Svénsen, B. (1994): Practical Lexicography, Oxford, Oxford University Press.

Lombez, C. (2003): Transactions secrètes. Philippe Jaccottet, poète et traducteur de Rilke et Hölderlin, Arras, Artois Presses Université, 182 p.

Par quelle mystérieuse alchimie deux poètes allemands des siècles passés, d'une part, Friedrich Hölderlin (1770-1843), et, d'autre part, Rainer Maria Rilke (1875-1926), ont-ils pu marquer d'une empreinte indélébile l'écriture fictionnelle du poète vaudois contemporain Philippe Jaccottet? Réponse: par la traduction française de ces deux auteurs, que le Suisse a pratiqué tout au long de son propre parcours littéraire. L'impact des œuvres traduites sur ses créations personnelles est considérable; en effet, sa création plonge des racines profondes dans le «terreau intellectuel» qu'il a su mettre au jour dans les écrits, comme le formule l'auteure.

Christine Lombez, normalienne, agrégée et docteur, nous livre dans son ouvrage Transactions secrètes, (c'est le titre, mis au pluriel d'un recueil de textes de Jaccottet, paru en 1987), les résultats d'une étude très fouillée sur la nature complexe des échanges «secrets» qui s'opèrent entre l'œuvre créatrice du poète - traducteur suisse et ses traductions de Rilke et Hölderlin. De tout temps, les poètes ont entretenu un rapport privilégié avec la traduction: les liens qui existent entre les deux activités, sans avoir été pour autant à chaque fois formalisés, sont au cœur d'une même démarche.

L'auteure met en perspective l'étroite relation dialogique du poète créateur et des œuvres traduites et recherche les traces objectives laissées dans l'écriture poétique par les auteurs traduits. Elle met en lumière à la fois Jaccottet traducteur, ses conceptions de la traduction et la place que celle-ci occupe dans ses procédés créatifs.

Dans un chapitre liminaire très dense, l'auteure rappelle à grand renfort de citations, trop souvent de seconde main (via A. Berman), les controverses séculaires autour du littéralisme, des belles infidèles, et les apports de l'école allemande de traduction. À l'inverse des traductions «enjolivantes» et "ethnocentriques» à la française, l'école allemande fit jouer à la traduction, à travers le concept de Bildung un rôle fondamental dans la formation de la langue.

La traduction comme transformation et transposition créatrice a pour la langue un effet à la fois enrichissant et dynamisant. Leur thèse se résume comme suit: seule la traduction permet un recul suffisant par rapport à la «vision interne globale» de l'œuvre. Se prévalant d'un nouveau rapport qui s'institue entre l'homme et le langage, la traduction devient, comme plus tard chez Heidegger, partie intégrante de la réflexion philosophique. Cette école fonde l'hypothèse suivant laquelle traduire le réel en mots pose les mêmes problèmes que traduire d'une langue à l'autre. 